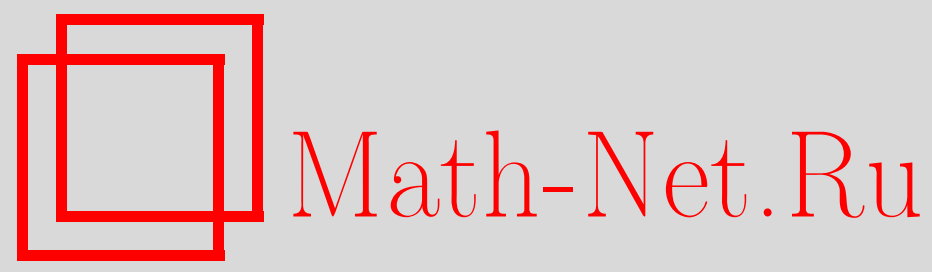

А. В. Олесов, О применении конформных отображений к неравенствам для тригонометрических полиномов, $\mathrm{Ma-}$ тем. заметки, 2004, том 76, выпуск 3, 396-408

DOI: https://doi.org/10.4213/mzm116

Использование Общероссийского математического портала Math-Net.Ru подразумевает, что вы прочитали и согласны с пользовательским соглашением http://www.mathnet.ru/rus/agreement

Параметры загрузки:

IP : 54.157 .27 .8

26 апреля 2023 г., 14:55:01

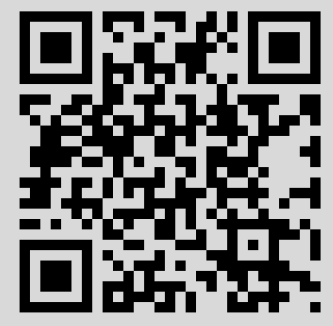




\section{О ПРИМЕНЕНИИ КОНФОРМНЫХ ОТОБРАЖЕНИЙ К НЕРАВЕНСТВАМ ДЛЯ ТРИГОНОМЕТРИЧЕСКИХ ПОЛИНОМОВ}

\section{А.В. Олесов}

В работе получены неравенства для тригонометрических и алгебраических полиномов, дополняющие и усиливающие классические результаты, восходящие к работам С. Н. Бернштейна и И. И. Привалова. Метод доказательства основан на построении по заданному тригонометрическому полиному конформного и однолистного отображения и на применении к этому отображению результатов геометрической теории функций комплексного переменного.

Библиограффия: 9 названий.

Введение. В данной работе устанавливается ряд точных неравенств для тригонометрических полиномов

$$
s_{n}(z)=\sum_{k=0}^{n}\left(a_{k} \cos k z+b_{k} \sin k z\right), \quad a_{k}, b_{k} \in \mathbb{R}, \quad a_{n}^{2}+b_{n}^{2} \neq 0
$$

удовлетворяющих условию

$$
\left|s_{n}(x)\right| \leqslant 1, \quad-\omega \leqslant x \leqslant \omega,
$$

при некотором $0<\omega<\pi$. В частности, для каждого комплексного $z$ указывается область, содержащая образ $s_{n}(z)$, ограниченная эллипсами и зависящая только от $z, \omega, n$ и коэффициентов $a_{n}, b_{n}$ (теорема 1 ). Получена оценка величины $\left|s_{n}^{\prime}(x)\right|$ на интервале $(-\omega, \omega)$, усиливающая результаты И. И. Привалова [1] и В. С. Виденского [2]. Эта теорема обобщает результат В.Н. Дубинина [3, с. 56] для всего периода и, тем самьм, представляет собой усиление классического неравенства Бернштейна-Сегё. Также усиливается и обобщается известньй результат для коэффициентов тригонометрических полиномов, а именно: тригонометрический полином вида (1) не может при всех вещественных значениях оставаться по модулю меньше, чем $\sqrt{a_{n}^{2}+b_{n}^{2}}$ (теорема 3 ). В качестве следствий основных теорем приводится ряд неравенств для алгебраических полиномов, дополняющих и усиливающих классические результаты, восходящие к работам С. Н. Бернштейна и П. Л. Чебьшева.

Работа выполнена при финансовой поддержке Российского фонда фундаментальных исследований, грант № 02-01-00028. 
В п. 1 данной статьи вводится ряд вспомогательных функций, среди которых конформное и однолистное отображение $w=f(\zeta)$, строяшееся по заданному тригонометрическому полиному и играющее в работе ключевую роль. В п. 2 приводятся основные результаты, полученные путем применения к указанному конформному отображению результатов геометрической теории функций комплексного переменного. Данньй подход к получению неравенств для полиномов был предложен Дубининым в работах [3], [4].

1. Некоторые конформные отображения. В этом пункте мы рассмотрим ряд вспомогательных аналитических функций и их свойства, которые нам понадобятся в дальнейшем.

Для фиксированного $\omega, 0<\omega<\pi$, определим функцию

$$
\xi=\varphi(w):=-i \sin \omega \Psi\left(i \frac{w+\sin (\omega / 2)}{\cos (\omega / 2)}\right)+\cos \omega, \quad|w|>1
$$

Здесь и ниже

$$
\Psi(w)=\frac{1}{2}\left(w+\frac{1}{w}\right)
$$

- функция Жуковского. Используя свойство функции Жуковского конформно и однолистно переводить внешность (а также внутренность) окружности, проходящей через точки \pm 1 на внешность соответствующей круговой дуги [5, с. 311], легко убедиться в том, что функция (3) конформно и однолистно отображает область $|w|>1$ на внешность дуги

$$
\bar{\gamma}=\left\{\xi=e^{i x}:-\omega \leqslant x \leqslant \omega\right\},
$$

причем бесконечность переходит в бесконечность. Напомним также, что при отображении функцией $\Psi(w)$ образом окружности $|w|=r$ является эллипс с фокусами в точках \pm 1 и большой осью равной $r+1 / r$.

Преобразуя (3), получим

$$
\varphi(w)=w \frac{1+w \sin (\omega / 2)}{w+\sin (\omega / 2)} .
$$

Подстановкой в (4) нетрудно проверить справедливость равенства

$$
\psi(\xi)=-\psi\left(\frac{1}{\xi}\right) \xi, \quad \xi \in \mathbb{C}_{\xi} \backslash\{\bar{\gamma} \cup\{0\}\},
$$

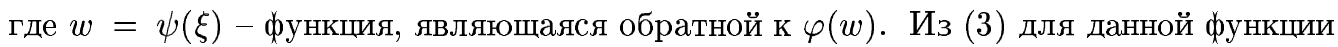
получим следующее представление:

$$
\psi(\xi)=-i \cos \left(\frac{\omega}{2}\right) \widetilde{\Phi}\left(i \frac{\xi-\cos \omega}{\sin \omega}\right)-\sin \left(\frac{\omega}{2}\right), \quad \xi \in \mathbb{C}_{\xi} \backslash \bar{\gamma}
$$

где регулярная ветвь функции $\widetilde{\Phi}(z)=z+\sqrt{z^{2}-1}$ выбирается на плоскости с круговым разрезом $\{z: z=i(\xi-\cos \omega) / \sin \omega, \xi \in \bar{\gamma}\}$ из условия $\widetilde{\Phi}(\infty)=\infty$.

Обозначим через $w=\Phi(z)$ функцию, определенную на внешности отрезка $[-1,1]$, являющуюся обратной к функции Жуковского $\Psi(w),|w|>1$. Из равенства $\Phi(z)=$ $z+\sqrt{z^{2}-1}$ находим

$$
\frac{\Phi^{\prime}(z)}{\Phi(z)}=\frac{1}{\sqrt{z^{2}-1}}
$$


Пусть тригонометрический полином $s_{n}(z)$ вида (1) удовлетворяет неравенству (2) и пусть

$$
Q_{n}(\xi)=\sum_{k=0}^{n}\left(a_{k}-i b_{k}\right) \xi^{k} .
$$

Поскольку $s_{n}(x)=\operatorname{Re} Q_{n}\left(e^{i x}\right),-\pi \leqslant x \leqslant \pi$, при всех комплексных $z$ имеет место равенство $s_{n}(z)=\rho\left(e^{i z}\right)$, в котором

$$
\rho(\xi)=\frac{1}{2}\left[Q_{n}(\xi)+\overline{Q_{n}\left(\frac{1}{\bar{\xi}}\right)}\right] .
$$

На множестве $G=\{w:|w|>1, \rho(\varphi(w)) \notin[-1,1]\}$ определим регулярную функцию $\zeta=F(w)$, полагая в точках $w, \varphi(w) \neq 0$,

$$
F(w)=w \frac{\Phi[\rho(\varphi(w))]}{\Phi^{n}[\delta(\varphi(w))]}
$$

где функция $\delta(\xi)$ зависит от параметра $0<\omega<\pi$ и определяется равенством

$$
\delta(\xi)=\frac{2}{1-\cos \omega} \Psi(\xi)-\frac{1+\cos \omega}{1-\cos \omega}
$$

Пусть $\mathfrak{D}$ - совокупность областей, составляющих множество $G \backslash\{w:|F(w)|=1\}$. В силу принщипа максимума модуля для регулярной функции

$$
\left|\frac{w}{\Phi[\delta(\varphi(w))]}\right|<1, \quad|w|>1 .
$$

Отсюда, а также из граничных свойств функции $\Phi[\rho(\varphi(w))]$ следует, что при приближении точки $w$ к границе каждой области из $\mathfrak{D}$ все предельные значения модуля $|F(w)|$ меньше либо равны единище. Более того, $0<\left|F^{\prime}(\infty)\right|<\infty$. Повторяя доказательство леммы $2.2[4]$ по отношению к функции $1 / F(1 / w)$, убеждаемся, что для любой области $D \in \mathfrak{D}$ вьполняется либо $F(D) \cap\{\zeta:|\zeta|>1\}=\varnothing$, либо $F(D)=\{\zeta:|\zeta|>1\}$. Во втором случае существует обратная к $F(w)$ функция $w=f(\zeta)$, однолистно отображающая $|\zeta|>1$ в область $D$.

Далее, через $T_{n}(z)$ обозначается $n$-й полином Чебьшева. Как известно,

$$
T_{n}(z)=\frac{1}{2}\left[\left(z+\sqrt{z^{2}-1}\right)^{n}+\left(z-\sqrt{z^{2}-1}\right)^{n}\right]
$$

Положим

$$
t_{n}(z)=T_{n}\left(\delta\left(e^{i z}\right)\right) .
$$

Ясно, что $t_{n}(x)$ - тригонометрический полином порядка $n$, удовлетворяющий условию (2). В дальнейшем он играет роль экстремального полинома.

2. Неравенства для полиномов. Всюду ниже приняты обозначения из п. 1. 
ТЕОРемА 1. Пусть тригонометрический полином $s_{n}(z)$ вида (1) удовлетворяет неравенству (2). Тогда для любого z вне системы отрезков $[-\omega+2 \pi k, \omega+2 \pi k]$, $k=0, \pm 1, \pm 2, \ldots$, имеет место неравенство

$$
\left|s_{n}(z)-1\right|+\left|s_{n}(z)+1\right| \leqslant 2 \Psi\left[\frac{\beta_{\lambda, r(z)}}{r(z)}\left|\Phi\left(t_{n}(z)\right)\right|\right] \leqslant\left|t_{n}(z)-1\right|+\left|t_{n}(z)+1\right| .
$$

В случае $z= \pm i \operatorname{Ln} \varphi(w),|w|>r_{\lambda}$, имеем

$$
\left|s_{n}(z)-1\right|+\left|s_{n}(z)+1\right| \geqslant 2 \Psi\left[\frac{\alpha_{\lambda, r(z)}}{r(z)}\left|\Phi\left(t_{n}(z)\right)\right|\right]>\left|t_{n-1}(z)-1\right|+\left|t_{n-1}(z)+1\right| .
$$

Здесь

$$
\lambda=\sqrt{a_{n}^{2}+b_{n}^{2}} \sin ^{2 n}\left(\frac{\omega}{2}\right), \quad r(z)=\left|\psi\left(e^{-i z \operatorname{sign}(\operatorname{Im} z)}\right)\right|,
$$

$\alpha_{\lambda, r(z)}, \beta_{\lambda, r(z)}-$ корни уравнений

$$
\lambda(r(z)+1)^{2} x=r(z)(x+1)^{2} \quad u \quad \lambda(r(z)-1)^{2} x=r(z)(x-1)^{2}
$$

соответственно, лежсащие на интервале $(1, r(z)]$;

$$
r_{\lambda}=2 \lambda^{-1}-1+2 \sqrt{\lambda^{-1}\left(\lambda^{-1}-1\right)} .
$$

Знаки равенства в любом из нестрогих неравенств (11), (12) достигаются только $\partial \Omega_{\text {я }} s_{n}(z)= \pm t_{n}(z)$.

ДокАЗАТЕЛЬСтво. Пусть $w=f(\zeta)$ - функция из п. 1. Непосредственньм вычислением получаем

$$
\frac{1}{f^{\prime}(\infty)}=\lim _{w \rightarrow \infty} \frac{F(w)}{w}=\left(a_{n}-i b_{n}\right) \sin ^{2 n}\left(\frac{\omega}{2}\right)
$$

Откуда с учетом леммы Шварца имеем $\left|f^{\prime}(\infty)\right|^{-1}=\lambda \leqslant 1$.

Функция $f^{*}(\zeta)=f^{\prime}(\infty) / f(1 / \zeta)$ в круге $|\zeta|<1$ однолистна, по модулю меньше, чем $\lambda^{-1}$, и представляется степенным рядом $f^{*}(\zeta)=\zeta+\alpha_{2} \zeta^{2}+\alpha_{3} \zeta^{3}+\cdots$ В классе таких функций известны точные оценки

$$
\left(\frac{1+\left|\lambda f^{*}(\zeta)\right|}{1+|\zeta|}\right)^{2} \leqslant\left|\frac{f^{*}(\zeta)}{\zeta}\right| \leqslant\left(\frac{1-\left|\lambda f^{*}(\zeta)\right|}{1-|\zeta|}\right)^{2}, \quad 0<|\zeta|<1
$$

Знак равенства слева или справа в (13), хотя бы в одной точке, имеет место тогда и только тогда, когда

$$
\frac{f^{*}(\zeta)}{\left[1+e^{i \alpha} \lambda f^{*}(\zeta)\right]^{2}} \equiv \frac{\zeta}{\left(1+e^{i \alpha} \zeta\right)^{2}}
$$

$\alpha$ вещественно (см., например, [4], [6]).

Пусть $w,|w|=r, \varphi(w) \neq 0$, есть точка множества $f(|\zeta|>1)$. Правое неравенство в (13) дает

$$
\frac{(|F(w)|-1)^{2}}{|F(w)|} \leqslant \lambda \frac{(r-1)^{2}}{r} .
$$


Ввиду строгого возрастания функции $y=(x-1)^{2} / x$ на промежутке $x>1$ существует единственньй корень $\beta_{\lambda, r}$ уравнения $\lambda(r-1)^{2} x=r(x-1)^{2}$, лежаший в интервале $(1, r]$. По этой же причине выполняется $|F(w)| \leqslant \beta_{\lambda, r}$, т.е.

$$
|\Phi[\rho(\varphi(w))]| \leqslant \frac{\beta_{\lambda, r}}{r}\left|\Phi^{n}[\delta(\varphi(w))]\right| \leqslant\left|\Phi^{n}[\delta(\varphi(w))]\right| .
$$

Если же $w \notin f(|\zeta|>1)$, то будет выполняться неравенство $|F(w)| \leqslant 1$, т.е.

$$
|\Phi[\rho(\varphi(w))]| \leqslant \frac{1}{r}\left|\Phi^{n}[\delta(\varphi(w))]\right|<\frac{\beta_{\lambda, r}}{r}\left|\Phi^{n}[\delta(\varphi(w))]\right| .
$$

Таким образом, (15) вьполняется при любом $w,|w|>1, \varphi(w) \neq 0$. Делая замену $\varphi(w)=e^{i z}$ и замечая, что в ввиду равенств $(9),(10)$

$$
t_{n}(z)=\Psi\left\{\Phi^{n}\left[\delta\left(e^{i z}\right)\right]\right\}
$$

убеждаемся в справедливости неравенств $(11)$ при $r(z)=\left|\psi\left(e^{i z}\right)\right|$. Переход при необходимости к тригонометрическому полиному $s_{n}(-z)$ позволяет в $(11)$ брать $r(z)=$ $\left|\psi\left(e^{-i z \operatorname{sign}(\operatorname{Im} z)}\right)\right|$, что имеет смысл в связи с убыванием дроби $\beta_{\lambda, r} / r$ при увеличении $r$ и ввиду равенства (5).

Убедимся теперь в справедливости неравенств (12). Пусть $w,|w|=r>r_{\lambda}, \varphi(w) \neq 0$, есть точка множества $f(|\zeta|>1)$. Левое неравенство в (13) и строгое возрастание функции $y=(x+1)^{2} / x$ на промежутке $x>1$ дают

$$
\frac{(|F(w)|+1)^{2}}{|F(w)|} \geqslant \lambda \frac{(r+1)^{2}}{r}>\lambda \frac{\left(r_{\lambda}+1\right)^{2}}{r_{\lambda}}=4 .
$$

Следовательно, существует единственный корень $\alpha_{\lambda, r}$ уравнения $\lambda(r+1)^{2} x=r(x+1)^{2}$, лежащий в интервале $(1, r]$, и для этого корня имеем

$$
|F(w)| \geqslant \alpha_{\lambda, r}
$$

Отсюда, а также из (8)

$$
|\Phi[\rho(\varphi(w))]| \geqslant \frac{\alpha_{\lambda, r}}{r}\left|\Phi^{n}[\delta(\varphi(w))]\right|>\left|\Phi^{n-1}[\delta(\varphi(w))]\right| .
$$

Покажем теперь, что любая точка $w,|w|>r_{\lambda}$, принадлежит образу $f(|\zeta|>1)$. Предположим от противного, что имеет место неравенство

$$
r_{\lambda}<r^{*}=\inf \{r: r>1,|F(w)|>1 \forall w,|w|=r\} .
$$

На окружности $|w|=r^{*}$ найдется точка $w^{*}$, удовлетворяющая условию

$$
\left|F\left(w^{*}\right)\right|=1 \text {. }
$$

С другой стороны, для любой последовательности $w_{k},\left|w_{k}\right|>r^{*}, k=1,2, \ldots$, сходящейся к $w^{*}$, из (17) получим

$$
\left|F\left(w_{k}\right)\right| \geqslant \alpha_{\lambda, r^{*}}, \quad k=1,2, \ldots,
$$


что противоречит условию (19). Таким образом, (18) имеет место при любом $w,|w|=$ $r>r_{\lambda}, \varphi(w) \neq 0$. Делая замену $\varphi(w)=e^{i z}$ и переходя при необходимости к тригонометрическому полиному $s_{n}(-z)$, убеждаемся в справедливости (12) при

$$
z= \pm i \operatorname{Ln} \varphi(w), \quad|w|>r_{\lambda}
$$

и $r(z)=|w|$. Согласно равенству (5), если точка $\xi,|\xi|<1$, принадлежит образу $\varphi\left(|w|>r_{\lambda}\right)$, то этому образу принадлежит также и $1 / \xi$. Следовательно, для любого $z$, удовлетворяющего условию $(20)$, величина $\left|\psi\left(e^{-\operatorname{sign}(\operatorname{Im} z) i z}\right)\right|$ больше, чем $r_{\lambda}$, и может быть принята за $r(z)$ в (12). В целесообразности такого выбора нас убеждает неравенство $\left|\psi\left(e^{i z \operatorname{sign}(\operatorname{Im} z)}\right)\right| \leqslant\left|\psi\left(e^{-i z \operatorname{sign}(\operatorname{Im} z)}\right)\right|$ и возрастание дроби $\alpha_{\lambda, r} / r$ с ростом $r$ на промежутке $r>r_{\lambda}$.

Приступим к доказательству случая равенства. Докажем сначала, что в случае $\lambda<1$ функция $f^{*}(\zeta)$ не может удовлетворять тождеству (14). Действительно, предполагая противное, получим

$$
\Psi\left(e^{-i \alpha} F(w)\right) \equiv \lambda \Psi\left(e^{i \beta} w\right)+\lambda-1, \quad \beta=\arg \left(a_{n}-i b_{n}\right)-\alpha, \quad w \in G
$$

Отсюда

$$
\rho(\varphi(w))=\Psi\left[w^{-1} \Phi^{n}[\delta(\varphi(w))] e^{i \alpha} \Phi\left(\lambda \Psi\left(e^{i \beta} w\right)+\lambda-1\right)\right] .
$$

Поскольку $\Phi(z)$ двузначна в точках отрезка $(-1,1)$ и принимает на нем значения, равные по модулю единице, то учитьвая (8), а также однолистность функции Жуковского во внешности единичного круга, заключаем, что функция, стоящая справа в последнем равенстве, не может быть однозначно и регулярно определена в точках $w=r e^{-i \beta}$, $1<r<\Phi\left(2 \lambda^{-1}-1\right)$, но это противоречит определениям функций, стоящих слева.

Если $\lambda=1$, то согласно лемме Шварца $f^{*}(\zeta) \equiv \zeta$, при этом неравенства (13) превращаются в равенства. Поскольку равенство справа в (15) возможно только при $\lambda=1$, заключаем, что знаки равенства в (15) и слева в (18) имеют место лишш при $F(w) \equiv \eta w$, $|\eta|=1$, откуда

$$
s_{n}(z)=\Psi\left\{\eta \Phi^{n}\left[\delta\left(e^{i z}\right)\right]\right\}
$$

но функция, стоящая справа в последнем равенстве, вещественна на $(\pi-\omega, \pi+\omega)$ только при $\eta= \pm 1$. Отсюда с учетом (16) следует равенство $s_{n}(z)= \pm t_{n}(z)$. Теорема доказана.

ЗАмЕЧАниЕ 1. В условиях теоремы 1 неравенство (12) справедливо для всех $z$, удовлетворяющих условию

$$
|\operatorname{Im} z|>\ln \left(r_{\lambda} \frac{1+r_{\lambda} \sin (\omega / 2)}{r_{\lambda}+\sin (\omega / 2)}\right) .
$$

Действительно, используя представление (4), нетрудно показать, что

$$
\varphi\left(r_{\lambda}\right)=\max \left\{|\varphi(w)|:|w|=r_{\lambda}\right\}
$$

после чего остается заметить, что область $|\xi|>\varphi\left(r_{\lambda}\right)$ лежит в образе $\varphi\left(|w|>r_{\lambda}\right)$. 
СлЕДСТВИЕ 1. Если тригонометрический полином $s_{n}(z)$ вида (1) удовлетворяет неравенству

$$
\left|s_{n}(x)\right| \leqslant 1, \quad-\pi \leqslant x \leqslant \pi
$$

то для любого $r>1$ и любого $z,|\operatorname{Im} z|=\ln r$, выполняется

$$
\left|s_{n}(z)-1\right|+\left|s_{n}(z)+1\right| \leqslant 2 \Psi\left(\beta_{\lambda, r} r^{n-1}\right) \leqslant 2 \Psi\left(r^{n}\right) .
$$

В случае $r>2 \lambda^{-1}-1+2 \sqrt{\lambda^{-1}\left(\lambda^{-1}-1\right)}$ имеем

$$
\left|s_{n}(z)-1\right|+\left|s_{n}(z)+1\right| \geqslant 2 \Psi\left(\alpha_{\lambda, r} r^{n-1}\right)>2 \Psi\left(r^{n-1}\right) .
$$

Здесь $\lambda=\sqrt{a_{n}^{2}+b_{n}^{2}} ; \beta_{\lambda, r}-$ корень уравнения

$$
\lambda(r-1)^{2} x=r(x-1)^{2}, \quad 1<\beta_{\lambda, r} \leqslant r
$$

$\alpha_{\lambda, r}-$ корень уравнения

$$
\lambda(r+1)^{2} x=r(x+1)^{2}, \quad 1<\alpha_{\lambda, r} \leqslant r .
$$

Для тригонометрических полиномов вида

$$
s_{n}(z)=a_{n} \cos n z+b_{n} \sin n z, \quad a_{n}^{2}+b_{n}^{2}=1,
$$

нестрогие неравенства в (22), (23) обращаются в равенства.

ДокАЗАтЕльство. Неравенства (22), (23) получаются из неравенств (11), (12) в результате предельного перехода $\omega \rightarrow \pi$. Для доказательства случая равенства проще всего заметить, что тригонометрический полином (26) можно представить в виде $s_{n}(z)=\Psi\left(\eta e^{i n z}\right),|\eta|=1$, после чего случай равенства проверяется непосредственно.

ЗАмЕЧАниЕ 2. Настоящий результат можно доказать по аналогии с теоремой 1 , рассматривая функцию леммы 2.2 работы [4]. Данный способ позволяет установить, что знаки равенства в $(22),(23)$ достигаются только для тригонометрических полиномов вида (26). Отметим также, что неравенство (22) эквивалентно теореме 3.1 работы [4], сформулированной в терминах алгебраических полиномов.

СЛЕДСТВИЕ 2. Если аләебраический полином

$$
P_{n}(z)=\sum_{k=0}^{n} c_{k} z^{k}, \quad c_{k} \in \mathbb{R}, \quad k=1,2, \ldots, \quad c_{n} \neq 0
$$

удовлетворяет неравенству

$$
\left|P_{n}(x)\right| \leqslant 1, \quad-1 \leqslant x \leqslant 1
$$

mo npu

$$
r>2 \lambda^{-1}-1+2 \sqrt{\lambda^{-1}\left(\lambda^{-1}-1\right)},
$$

образ эллипса $|z-1|+|z+1|=2 \Psi(r)$ при отображении $w=P_{n}(z)$, есть кривая, лежащая вне әллипса $|w-1|+|w+1|=2 \Psi\left(\alpha_{\lambda, r} r^{n-1}\right)$ и внутри әллипса $|w-1|+|w+1|=2 \Psi\left(\beta_{\lambda, r} r^{n-1}\right)$. Здесь $\lambda=\left|c_{n}\right| / 2^{n-1}, \alpha_{\lambda, r} u \beta_{\lambda, r}-$ корни уравнений (25) и (24) соответственно. Для полинома $T_{n}(z)$ указанные әллипсы совпадают. 
ДокАЗАТЕЛЬСТво следует из неравенств (22) и (23), примененных к тригонометрическому полиному $s_{n}(t)=P_{n}(\cos t)$. Случай равенства проверяется непосредственно.

Отметим, что настоящий результат при комплексных коэффициентах $P_{n}(z)$ неверен. Что касается оценки сверху, то из неравенств такого типа известно неравенство

$$
\left|P_{n}(z)\right| \leqslant r^{n}, \quad|z-1|+|z+1|=2 \Psi(r), \quad r>1,
$$

справедливое для любых полиномов, удовлетворяющих (28). Этот результат был установлен Бернштейном на основании принципа максимума [7, с. 74].

Перейдем к вопросу об экстремальной оценке производной тригонометрического полинома на отрезке меньшем, чем период. В монографии [1] Привалов установил, что если тригонометрический полином $s_{n}(z)$ порядка $n$ удовлетворяет неравенству (2), то

$$
\left|s_{n}^{\prime}(x)\right| \leqslant C_{\omega, \varepsilon} n, \quad-\omega+\varepsilon \leqslant x \leqslant \omega-\varepsilon, \quad 0<\varepsilon<\frac{\omega}{2},
$$

где $C_{\omega, \varepsilon}-$ постоянная, зависящая от $\omega$ и $\varepsilon$. Виденский [2] уточнил этот результат, показав, что (2) влечет за собой неравенство

$$
\left|s_{n}^{\prime}(x)\right| \leqslant n \cos \left(\frac{x}{2}\right)\left[\sin ^{2}\left(\frac{\omega}{2}\right)-\sin ^{2}\left(\frac{x}{2}\right)\right]^{-1 / 2}, \quad-\omega<x<\omega .
$$

\section{Имеет место следующая}

ТЕОРема 2. Если тригонометрический полином $s_{n}(z)$ вида (1) удовлетворяет неравенству (2), то

$$
\begin{aligned}
\left|s_{n}^{\prime}(x)\right| \leqslant & \frac{\cos (x / 2) \sqrt{1-s_{n}^{2}(x)}}{\sqrt{\sin ^{2}(\omega / 2)-\sin ^{2}(x / 2)}} \\
& \times\left[n-\frac{\Lambda(\omega, x) \cos (\omega / 2)\left(1-\sin ^{n}(\omega / 2)\left(a_{n}^{2}+b_{n}^{2}\right)^{1 / 4}\right)}{2 \cos (x / 2)}\right], \quad-\omega<x<\omega,
\end{aligned}
$$

$2 \partial e$

$$
\Lambda(\omega, x)=\left|\Phi\left(i \frac{e^{i x}-\cos \omega}{\sin \omega}\right)\right| .
$$

Для тригонометрического полинома $t_{n}(z)$ неравенство (29) обращается в равенство.

ДоКАЗАТЕЛЬСТВО. Пусть функция $w=f(\zeta)$ из п. 1 . Если точка $\zeta,|\zeta|=1$, является точкой регулярности функции $f(\zeta)$, причем $|f(\zeta)|=1$, то из правой части (13) предельным переходом получим

$$
\left|f^{\prime}(\zeta)\right| \geqslant \sin ^{-n}\left(\frac{\omega}{2}\right)\left(a_{n}^{2}+b_{n}^{2}\right)^{-1 / 4}
$$

Если точка $w,|w|=1$, является точкой регулярности функции $F(w)$ и одновременно лежит на границе области $D \in \mathfrak{D}$ такой, что $F(D) \cap\{\zeta:|\zeta|>1\}=\varnothing$, то необходимо в этой точке

$$
\frac{\partial|F|}{\partial|w|} \leqslant 0
$$


Если $F(D)=\{\zeta:|\zeta|>1\}$, то, применяя неравенство $(30)$, заключаем, что в такой точке

$$
\frac{\partial|F|}{\partial|w|}=\left|f^{\prime}(\zeta)\right|^{-1} \leqslant \sin ^{n}\left(\frac{\omega}{2}\right)\left(a_{n}^{2}+b_{n}^{2}\right)^{1 / 4} .
$$

Таким образом, неравенство (31) вьполняется во всех точках единичной окружности за исключением, быть может, конечного числа таких точек.

Далее, под значениями функции $w=\psi(\xi)$ в точках дуги

$$
\gamma=\left\{\xi=e^{i x}:-\omega<x<\omega\right\}
$$

будем понимать значения, получаемые в результате регулярного продолжения этой функции из области $|\xi|>1$. В точках $w \in \psi(\gamma)$, в которые функции $\Phi[\rho(\varphi(w))], \Phi[\delta(\varphi(w))]$ конформно продолжаются из $|w|>1$, имеем

$$
\frac{\partial|F|}{\partial|w|}=1+\left|\frac{\partial}{\partial w} \Phi[\rho(\varphi(w))]\right|-\left|\frac{\partial}{\partial w} \Phi^{n}[\delta(\varphi(w))]\right|
$$

Полагая $\varphi(w)=e^{i x}$ и учитывая $(31)$, приходим к неравенству

$$
\begin{aligned}
& \left|\Phi^{\prime}\left[s_{n}(x)\right] s_{n}^{\prime}(x) \varphi^{\prime}\left(\psi\left(e^{i x}\right)\right)\right| \\
& \quad \leqslant n\left|\Phi^{\prime}\left[\delta\left(e^{i x}\right)\right] \delta^{\prime}\left(e^{i x}\right) \varphi^{\prime}\left(\psi\left(e^{i x}\right)\right)\right|-\left[1-\sin ^{n}\left(\frac{\omega}{2}\right)\left(a_{n}^{2}+b_{n}^{2}\right)^{1 / 4}\right],
\end{aligned}
$$

откуда после простых преобразований с учетом (7) получаем

$$
\frac{\left|s_{n}^{\prime}(x)\right|}{\sqrt{1-s_{n}^{2}(x)}} \leqslant n \frac{\cos (x / 2)}{\sqrt{\sin ^{2}(\omega / 2)-\sin ^{2}(x / 2)}}-\frac{1-\sin ^{n}(\omega / 2)\left(a_{n}^{2}+b_{n}^{2}\right)^{1 / 4}}{\left|\varphi^{\prime}\left(\psi\left(e^{i x}\right)\right)\right|} .
$$

Из (3) и (6) находим

$$
\varphi^{\prime}\left(\psi\left(e^{i x}\right)\right)=\sin \left(\frac{\omega}{2}\right)\left[1-\widetilde{\Phi}^{-2}\left(i \frac{e^{i x}-\cos \omega}{\sin \omega}\right)\right]
$$

откуда не составляет труда убедиться в справедливости равенства

$$
\left|\varphi^{\prime}\left(\psi\left(e^{i x}\right)\right)\right|=2\left|\widetilde{\Phi}\left(i \frac{e^{i x}-\cos \omega}{\sin \omega}\right)\right|^{-1} \frac{\sqrt{\sin ^{2}(\omega / 2)-\sin ^{2}(x / 2)}}{\cos (\omega / 2)} .
$$

Для доказательства неравенства (29) остается заметить, что при данном выборе точек $w$ на окружности $|w|=1$ выполняется

$$
\left|\widetilde{\Phi}\left(i \frac{e^{i x}-\cos \omega}{\sin \omega}\right)\right|=\Lambda(\omega, x) .
$$

Случай равенства следует из тождества $F(w) \equiv w$, справедливого для указанных тригонометрических полиномов. Теорема доказана. 
ЗАмЕчАниЕ 3. Настоящий результат доказан для вещественных тригонометрических полиномов. Если $s_{n}(z)$ - тригонометрический полином с комплексными коэффициентами, то для каждого $x \in(-\omega, \omega)$ замена $s_{n}(x)$ на $e^{i \theta} s_{n}(x), \theta=-\arg s^{\prime}(x)$, и подстановка в (29) приводит к усилению неравенства Виденского за счет уменьшения множителя $n$.

СлЕДСТВИЕ 3. Если тригонометрический полином $s_{n}(z)$ вида (1) удовлетворяет неравенству (21), то

$$
\left|s_{n}^{\prime}(x)\right| \leqslant\left(n-1+\left(a_{n}^{2}+b_{n}^{2}\right)^{1 / 4}\right) \sqrt{1-s_{n}^{2}(x)}, \quad-\pi \leqslant x \leqslant \pi .
$$

Для доказательства достаточно перейти к пределу $\omega \rightarrow \pi$ в правых частях (32) и (33).

Это неравенство впервые установлено Дубининым в статье [3] и представляет собой усиление неравенства Бернштейна-Сегё.

Хорошо известно замечательное неравенство Бернштейна $[7$, с. 168] об оценке производной алгебраического полинома $P_{n}(x)$ степени не вьше $n$ внутри данного интервала. Оно имеет вид

$$
\left|P_{n}^{\prime}(x)\right| \sqrt{1-x^{2}} \leqslant n \max _{-1 \leqslant x \leqslant 1}\left|P_{n}(x)\right|, \quad-1 \leqslant x \leqslant 1 .
$$

Следствие 3 позволяет усилить это неравенство следующим образом.

СлЕДСТВИЕ 4. Если алгебраический полином вида (27) удовлетворяет неравенству (28), то

$$
\left|P_{n}^{\prime}(x)\right| \sqrt{1-x^{2}} \leqslant n-1+\sqrt{\frac{\left|c_{n}\right| \sqrt{1-T_{n}^{2}(x)}}{2^{n-1}}}, \quad-1 \leqslant x \leqslant 1
$$

Равенство достигается для полинома $T_{n}(z)$ в точках, являющихся его нулями.

Для доказательства достаточно применить неравенство (34) к тригонометрическому полиному

$$
s_{n}(t)=\frac{1}{2}\left[P_{n}\left(\cos \left(t_{0}+t\right)\right)-P_{n}\left(\cos \left(t_{0}-t\right)\right)\right], \quad t_{0}=\arccos x,
$$

и положить $t=0$. Случай равенства проверяется непосредственно.

СлЕДСТВИЕ 5. Если тригонометрический полином $s_{n}(z)$ вида (1) удовлетворяет неравенству (21) и $s_{n}(x)=0$ при $x=0, \pi$, то

$$
\left|\frac{s_{n}(x)}{\sin x}\right| \leqslant n-1+\left(a_{n}^{2}+b_{n}^{2}\right)^{1 / 4}, \quad-\pi \leqslant x \leqslant \pi .
$$

Знак равенства достигается для $s_{n}(z)=\sin n z$ в точках $x=0, \pi$. 
ДокАЗАТЕЛЬСТво. Нетрудно показать, что на вещественной оси экстремум дроби $s_{n}(x) / \sin x$ достигается при $\operatorname{tg} x=s_{n}(x) / s_{n}^{\prime}(x)$, т.е. при $\sin x=s_{n}(x) / \sqrt{s_{n}^{2}(x)+s_{n}^{\prime 2}(x)}$, поэтому он не превышает максимума $\sqrt{s_{n}^{2}(x)+s_{n}^{\prime 2}(x)}$. В свою очередь, из (34) легко вывести неравенство

$$
\sqrt{s_{n}^{2}(x)+s_{n}^{\prime 2}(x)} \leqslant n-1+\left(a_{n}^{2}+b_{n}^{2}\right)^{1 / 4} .
$$

Случай равенства проверяется непосредственно.

ТЕОРЕМа 3. Если тригонометрический полином $s_{n}(z)$ порядка $n \geqslant 2$ вида (1) удовлетворяет неравенству (2), то

$$
\sqrt{a_{n}^{2}+b_{n}^{2}} \sin ^{2 n} \frac{\omega}{2}\left[1+\frac{1}{\sin (\omega / 2)}\left|\frac{a_{n-1}+i b_{n-1}}{2\left(a_{n}+i b_{n}\right)}+n \cos ^{2} \frac{\omega}{2}\right|\right] \leqslant 1 .
$$

Равенство имеет место лишь для полиномов $s_{n}(z)= \pm t_{n}(z)$.

ДоКАЗАТЕЛЬСТВо. В обозначениях п. 1 пусть в некоторой проколотой окрестности точки $w=0$

$$
\widetilde{F}(w):=\frac{1}{F(1 / w)} \equiv w \frac{\Phi^{n}[\delta(\varphi(1 / w))]}{\Phi[\rho(\varphi(1 / w))]}
$$

и пусть $\Delta(w)=w \widetilde{F}^{\prime}(w) / \widetilde{F}(w)$. Для последней функции имеем

$$
\Delta(w)=1+\frac{\varphi^{\prime}(1 / w)}{w}\left[\frac{\Phi^{\prime}[\rho(\xi)] \rho^{\prime}(\xi)}{\Phi[\rho(\xi)]}-n \frac{\Phi^{\prime}[\delta(\xi)] \delta^{\prime}(\xi)}{\Phi[\delta(\xi)]}\right], \quad \xi=\varphi\left(\frac{1}{w}\right) .
$$

Заметим, что $w \varphi(1 / w) \rightarrow \varphi^{\prime}(\infty)=\sin (\omega / 2)$ при $w \rightarrow 0$. Учитывая данный факт, а также равенство (7), получим

$$
\begin{aligned}
& \lim _{w \rightarrow 0} \frac{\Delta(w)-1}{w}=\lim _{\xi \rightarrow \infty} \frac{\xi^{2}}{\sin (\omega / 2)}\left[\frac{\rho^{\prime}(\xi)}{\sqrt{\rho^{2}(\xi)-1}}-n \frac{\delta^{\prime}(\xi)}{\sqrt{\delta^{2}(\xi)-1}}\right] \\
& =\lim _{\xi \rightarrow \infty} \frac{4 \sin (\omega / 2)}{c_{n} \xi^{n}}\left[\frac{1}{2}\left(Q_{n}^{\prime}(\xi) \xi-\frac{\overline{Q_{n}^{\prime}(1 / \bar{\xi})}}{\xi}\right)\left(\delta(\xi)-\left[\delta(\xi)-\sqrt{\delta^{2}(\xi)-1}\right]\right)\right. \\
& \left.-\frac{n(\xi-1 / \xi)}{2 \sin ^{2}(\omega / 2)}\left(\rho(\xi)-\left[\rho(\xi)-\sqrt{\rho^{2}(\xi)-1}\right]\right)\right] \\
& =\lim _{\xi \rightarrow \infty} \frac{4 \sin (\omega / 2)}{c_{n} \xi^{n}}\left[\frac{Q_{n}^{\prime}(\xi) \xi}{2}\left(\frac{\xi+1 / \xi}{2 \sin ^{2}(\omega / 2)}-\operatorname{ctg}^{2} \frac{\omega}{2}\right)\right. \\
& \left.-\frac{n \xi}{4 \sin ^{2}(\omega / 2)}\left(Q_{n}(\xi)+\overline{Q_{n}\left(\frac{1}{\bar{\xi}}\right)}\right)\right] \\
& =\lim _{\xi \rightarrow \infty} \frac{Q_{n}^{\prime}(\xi)\left(\xi-2 \cos ^{2}(\omega / 2)\right)-n Q_{n}(\xi)}{\sin (\omega / 2) c_{n} \xi^{n-1}} \\
& =\frac{-c_{n-1}-2 n \cos ^{2}(\omega / 2) c_{n}}{\sin (\omega / 2) c_{n}},
\end{aligned}
$$


где $c_{k}=a_{k}-i b_{k}, k=n-1, n$. С другой стороны, используя правило Лопиталя, легко показать, что

$$
\lim _{w \rightarrow 0} \frac{\Delta(w)-1}{w}=\frac{\widetilde{F}^{\prime \prime}(0)}{2 \widetilde{F}^{\prime}(0)}
$$

откуда

$$
\widetilde{F}^{\prime \prime}(0)=2 \widetilde{F}^{\prime}(0) \frac{-c_{n-1}-2 n \cos ^{2}(\omega / 2) c_{n}}{\sin (\omega / 2) c_{n}} .
$$

Обозначим через $\widetilde{f}(\zeta)$ функцию, однолистную в единичном круге $|\zeta|<1$, являющуюся обратной к $\widetilde{F}(w)$. Для этой функции

$$
\widetilde{f}^{\prime \prime}(0)=-\widetilde{F}^{\prime \prime}(0)\left(\tilde{f}^{\prime}(0)\right)^{3}=2 \frac{c_{n-1}+2 n \cos ^{2}(\omega / 2) c_{n}}{\sin (\omega / 2) c_{n}}\left(a_{n}-i b_{n}\right)^{2} \sin ^{4 n}\left(\frac{\omega}{2}\right) .
$$

Тогда при $\lambda=\sqrt{a_{n}^{2}+b_{n}^{2}} \sin ^{2 n}(\omega / 2)$ функция $f^{*}(\zeta)=\widetilde{f}(\zeta) / \tilde{f}^{\prime}(0)$ в круге $|\zeta|<1$ однолистна, по модулю меньше, чем $\lambda^{-1}$, и представляется степенным рядом

$$
f^{*}(\zeta)=\zeta+\alpha_{2} \zeta^{2}+\alpha_{3} \zeta^{3}+\cdots
$$

где $\alpha_{2}=\left[c_{n-1}+2 n \cos ^{2}(\omega / 2) c_{n}\right] \sin ^{2 n-1}(\omega / 2)$. В этом случае согласно [8, с. 94]

$$
\left|\alpha_{2}\right| \leqslant 2(1-\lambda)
$$

что эквивалентно неравенству (35), примененному к $s_{n}(-z)$.

Для доказательства случая равенства отметим, что равенство в (36) (см. [8, с. 94]) возможно лишш в случае вьполнения тождества (14), но, как было показано при доказательстве теоремы 1 , функция $f^{*}(\zeta)$ удовлетворяет указанному тождеству только при $s_{n}(z)= \pm t_{n}(z)$. Теорема доказана.

СлЕДСТВИЕ 6. Если алгебраический полином вида (27) удовлетворяет неравенству (28), mo

$$
\left|c_{n}\right|+\left|c_{n-1}\right| \leqslant 2^{n-1}
$$

Для полинома $T_{n}(z)$ достигается равенство.

Для доказательства в случае $n \geqslant 2$ достаточно применить (35) к тригонометрическому полиному $s_{n}(t)=P_{n}(\cos t)$. При $n=1$ справедливость (37) очевидна. Случай равенства проверяется непосредственно.

Интересно заметить, что неравенство В. А. Маркова [9]

$$
\left|P_{n}^{(k)}(x)\right| \leqslant T_{n}^{(k)}(1), \quad-1 \leqslant x \leqslant 1,
$$

справедливое в условиях следствия при $k=n-1$ эквивалентно неравенству

$$
\left|c_{n}\right|+\frac{\left|c_{n-1}\right|}{n} \leqslant 2^{n-1} .
$$


ЗАмЕчАниЕ 4. Можно показать, рассматривая функцию леммы 2.3 работы [4] и анализируя случай равенства в (36), что знак равенства в (37) при $n \geqslant 2$ имеет место только для полиномов $\pm T_{n}(z)$. Отметим также, что, используя разложение соответствующей функции $\Delta(w)$ в ряд Тейлора в окрестности точки $w=0$, не составляет труда получить рекуррентные формулы для вычисления первых $n+1$ коэффициентов степенного ряда функции указанной леммы. В связи с этим, можно применить неравенства для коэффициентов разложения соответствующего конформного отображения $f^{*}$, отличные от (36); в частности, применение неравенства $\left|\alpha_{2}^{2}-\alpha_{3}\right| \leqslant 1-\lambda^{2}[8$, с. 94] приводит в условиях следствия к точному неравенству

$$
c_{n}^{2}+\left|n c_{n}^{2}+4 c_{n} c_{n-2}\right| \leqslant 4^{n-1}, \quad n \geqslant 2 .
$$

Выражаю глубокую благодарность В. Н. Дубинину за внимательное отношение к моей работе.

\section{СПИСОК ЦИТИРОВАННОЙ ЛИТЕРАТУРЫ}

[1] Привалов И. И. Интеграл Коши. Саратов, 1919.

[2] Виденский В.С. Экстремальные оценки производной тригонометрического полинома на отрезке, меньшем чем период // Докл. АН СССР. 1960. Т. 130. №1. С. 13-16.

[3] Дубинин В.Н. Теоремы искажения для полиномов на окружности // Матем. сб. 2000. Т. 191. № 12. С. 51-60.

[4] Дубинин В. Н. Конформные отображения и неравенства для алгебраических полиномов // Алгебра и анализ. 2001. Т. 13. № 5. С. 16-43.

[5] Сидоров Ю. В., Федорюк М.В., Шабунин М.И. Лекции по теории функций комплексного переменного. М.: Наука, 1989.

[6] Лебедев Н. А. Некоторые оценки для функций регулярных и однолистных в круге // Вестн. ЛГУ. Сер. матем., физ., хим. 1955. №4. С. 3-21.

[7] Бернштейн С.Н. Экстремальные свойства полиномов и наилучшее приближение. М.-Л.: ОНТИ, 1937.

[8] Лебедев Н. А. Принцип площадей в теории однолистных функций. М.: Наука, 1975.

[9] Марков В.А. О функциях, наименее уклоняющихся от нуля в данном промежутке. СПб., 1892.

Морской государственный университет им. Г. И. Невельского, г. Владивосток 